REVTEX4-1

\title{
Geometric Momentum: the Proper Momentum for a Free Particle on a Two-dimensional Sphere
}

\author{
Q. H. Liu,* L. H. Tang, and D. M. Xun \\ School for Theoretical Physics, and Department of Applied Physics, \\ Hunan University, Changsha, 410082, China
}

(Dated: October 30, 2018)

\begin{abstract}
In Dirac's canonical quantization theory on systems with second-class constraints, the commutators between the position, momentum and Hamiltonian form a set of algebraic relations that are fundamental in construction of both the quantum momentum and the Hamiltonian. For a free particle on a two-dimensional sphere or a spherical top, results show that the well-known canonical momentum $p_{\theta}$ breaks one of the relations, while three components of the momentum expressed in the three-dimensional Cartesian system of axes as $p_{i}(i=1,2,3)$ are satisfactory all around. This momentum is not only geometrically invariant but also self-adjoint, and we call it geometric momentum. The nontrivial commutators between $p_{i}$ generate three components of the orbital angular momentum; thus the geometric momentum is fundamental to the angular one. We note that there are five different forms of the geometric momentum proposed in the current literature, but only one of them turns out to be meaningful.
\end{abstract}

PACS numbers: 03.65.Fd, 11.10.Ef 


\section{INTRODUCTION}

Although the fundamental principles of quantum constrained dynamics were outlined by Dirac in the 1950 s to $1960 \mathrm{~s}^{1}$, no consensus regarding the formulation has so far been reached when applied to particular systems of second-class constraints ${ }^{2-6}$. A subject of intense debate for decades has been: what the proper definition of momentum in quantum mechanics is for a free particle on a two-dimensional sphere. To obtain an unambiguous conclusion, we carefully analyze the geometric momentum (GM), which will be explicitly defined shortly. From the viewpoint of the GM, there are more than five different understandings, and each has its own exclusive conclusions from which we can see how physics proceeds with excitement, conflict, and controversy ${ }^{4}$. In this paper, we do not extensively deal with sphere $S^{N}$ in any $(N+1)$-dimensional flat space unless $N=2$, and this line of reasoning is applicable for sphere $S^{N}$ when $N \geq 2$.

According to Dirac, the canonical quantization procedure is an effective recipe of quantizing a classical theory while attempting to preserve the formal structure of the canonical Poisson brackets, or the Dirac brackets for a constrained system, to the extent possible ${ }^{1,7}$. However, from the very beginning, Dirac was well aware of the difficulty of the procedure, and he commented on it in a footnote of his Principles ${ }^{7}$, stressing that it is fundamentally correct only in Cartesian coordinates. Some think that the phrase Cartesian coordinates used by Dirac actually implies the Cartesian space, i.e., the flat space ${ }^{8-30}$. If so, what is the quantization procedure is developed in flat space, where the two-dimensional curved surface is embedded? Basically, investigations have been performed from two perspectives, and ours in the present work belongs to the second. The first is solely from the intrinsic point of view, dealing with quantities such as the distance element square $d s^{2}=g_{\mu v} d x^{\mu} d x^{v}$ within the surface as measured along curves on the surface parametrized by $\left(x^{\mu}, x^{v}\right)$, the LaplaceBeltrami operator $\nabla^{2}=1 / \sqrt{g} \partial_{\mu} g^{\mu v} \sqrt{g} \partial_{v}$, and the Gaussian curvature $K$. This approach allows mapping from the surface to a flat space, e.g., gnomonic projection ${ }^{31}$, multivalued mapping ${ }^{32,33}$ etc $^{34}$. The second is from both intrinsic and extrinsic points of view, where the presence of the mean curvature $H$, defined via the surface's embedding in a flat space, is a salient feature. In differential geometry, a two-dimensional surface cannot be completely specified unless two curvatures are known. How the constrained motion on the surface is advanced from the second prospective in recent decades will be discussed next. 


\section{A. Quantization in flat space and geometric potential and geometric momentum}

When a particle moves on a two-dimensional curved surface, we examine it in threedimensional flat space rather than on the surface itself. Moreover, because no geometric surface of zero thickness exists in the realistic world, it is better to conceive of a surface as a limiting case of a curved shell of equal thickness $z_{0}$, where the limit $z_{0} \rightarrow 0$ is then taken. Roughly speaking, there is a noninterchangeability of computational order: first taking limit $z_{0} \rightarrow 0$ and then defining the derivatives on the surface and first taking derivatives in bulk and then letting $z_{0} \rightarrow 0$. This observation sheds new light on the quantization of the kinetic energy $T=p^{2} /(2 \mu)$ and the momentum operator $\mathbf{p}$, which are reviewed with comments in the following.

Progress on the quantization of the kinetic energy was first initiated by Jensen, Koppe in $1971^{35}$, and was finally completed by da Costa in $1981^{36}$. Jensen, Koppe, and da Costa developed the so-called confining potential approach in which the two-dimensional curved surface is realized by limiting the three-dimensional system originally defined in flat space, and they found that the correct result of the quantum kinetic energy is

$$
T=-\frac{\hbar^{2}}{2 \mu} \nabla^{2}-\frac{\hbar^{2}}{2 \mu}\left(H^{2}-K\right) .
$$

This is a striking finding, and it is not only a gem in quantum mechanics but also a treasure in surface mathematics. Eq. (1) implies that the original Laplace-Beltrami operator $\nabla^{2}$ on the surface may not be enough unless a term $\left(H^{2}-K\right)$ is included,

$$
\nabla^{2} \rightarrow \nabla^{2}+\left(H^{2}-K\right)
$$

When an electromagnetic wave transmits through thin-layer media as the thickness is negligible, the curvature-dependent part $\left(H^{2}-K\right)$ of the Laplace-Beltrami operator can produce observable effects ${ }^{37}$. In quantum mechanics, the constraint-induced curvature-dependent potential $-\hbar^{2} /(2 \mu)\left(H^{2}-K\right)$ was simply called the geometric potential ${ }^{37}$, and it is attractive for $\left(H^{2}-K\right) \geq 0$. Because $H^{2}-K=0$ for the $S^{2}$ sphere, the Hamiltonian for a point particle freely moving on the surface still assumes the usual form as $H=T=-\hbar^{2} \nabla^{2} /(2 \mu)$, where $H$ should not be confused with the mean curvature. The confining potential procedure of quantization has been widely employed and investigated ever since ${ }^{38-50}$. From the experimental point of view, as many noted ${ }^{48}$, the realization of an optical analog of the 
curvature-induced geometric potential can be taken as empirical evidence for the validity of the confining potential procedure ${ }^{37}$.

As to the quantization of the momentum operator $\mathbf{p}=\left(p_{x}, p_{y}, p_{z}\right)$ expressed in the threedimensional Cartesian coordinate system $(x, y, z)$, a remarkable finding was made in $2007^{22}$, and the general form is given by

$$
\mathbf{p}=-i \hbar\left(\mathbf{r}^{\mu} \partial_{\mu}+H \mathbf{n}\right)
$$

where $\mathbf{r}=\left[x\left(x^{\mu}, x^{v}\right), y\left(x^{\mu}, x^{v}\right), z\left(x^{\mu}, x^{v}\right)\right]$ is the position vector on the surface, $\mathbf{r}^{\mu}=g^{\mu \nu} \mathbf{r}_{\nu}=$ $g^{\mu \nu} \partial \mathbf{r} / x^{\nu}$, and at this point $\mathbf{r}, \mathbf{n}=\left(n_{x}, n_{y}, n_{z}\right)$ denotes the normal and $H \mathbf{n}$ symbolizes the mean curvature vector field, a geometric invariant ${ }^{22}$. In contrast to the geometric potential we can call it GM because it in fact depends on the mean curvature. If simply denoting the gradient operator $\mathbf{r}^{\mu} \partial_{\mu}$ on the surface as $\nabla$, Eq. (3) implies the following correspondence:

$$
\nabla \rightarrow \nabla+H \mathbf{n}
$$

Quantities (1) and (3) assume their usual from, provided the surface is flat, $H=K=0$. This is good, but an immediate question arises: Can these new quantities be formulated into Dirac's theory for constrained motions? In the general case, this question is open, and we will give an answer in the near future. This paper focuses on an elaboration of the motion on a spherical surface where the answer is affirmative, as we see in sections III and VI.

\section{B. Comments on the routine paradigm of quantization on the curved surface}

The routine paradigm of quantization toward the motion on the surface is performed within intrinsic geometry irrespective of the existence of the higher-dimensional flat space in which the surface could be embedded. When applied to the motion of a point particle on two-dimensional surface, it is usually postulated ${ }^{51-53}$ that the Cartesian coordinate Laplacian should be replaced by the Laplace-Beltrami operator acting on this manifold and the kinetic energy is $T=-\hbar^{2} \nabla^{2} /(2 \mu)$ without the geometric potential. This is highly controversial and has been debated for decades. If the surface is a two-dimensional sphere $S^{2}$ of radius $r$, $T=-\hbar^{2} \nabla^{2} /(2 \mu)=L^{2} /\left(2 \mu r^{2}\right)$ where $L$ is the angular momentum of the particle. DeWitt studied the path-integral quantization of this problem and found an extra energy term proportional to the Gaussian curvature $1 / r^{2}$ in the Hamiltonian as $\alpha \hbar^{2} / r^{2}$, with $\alpha=1 / 24^{53}$. 
Others gave $\alpha=1 / 12^{54}$ and $\alpha=1 / 8^{55}$. These zero-point energies conflict with the cosmic observation $^{4,56}$ and must be discarded. Therefore, to deal with the quantization of the kinetic energy, on one hand, there is therefore no transparent and self-consistent way within this routine manner; on the other, as we understand today, the absence of the geometric potential is contradictory to the experiments. However, as to describing a free motion with curvilinear coordinates in the flat space, Podolsky's recipe gives for the kinetic energy $T=-\hbar^{2} \nabla^{2} /(2 \mu)^{51}$. This is, nevertheless, perfect. What is more, the quantum mechanics in flat space can be rewritten into curved space with a Maupertuis metric ${ }^{34}$, where the Laplace-Beltrami operator acquires an extra curvature term corresponding to a conformally invariant Laplace operator. In momentum space the quantum mechanics of the hydrogenatom is driven by a Laplace operator with yet another extra curvature term ${ }^{34}$. Conversely, with the help of a multivalued mapping it is possible to map the known flat-space physics into the curved-space physics with only the Laplace-Beltrami operator ${ }^{32,33}$.

\section{Previous utilization of Dirac's canonical quantization theory}

Let us turn to the Dirac's canonical quantization theory of a system with second-class constraints. Previous works find that various momentum including the usual canonical one and GM as well are all definable ${ }^{14,19}$. For a two-dimensional sphere ${ }^{13-30}$, the well-known canonical momenta $p_{\theta}=-i \hbar\left(\partial_{\theta}+\cot \theta / 2\right)$ and $p_{\varphi}=-i \hbar \partial_{\varphi}$ and the momenta (6)-(8) all seem to be permissible. In fact, neither of these momenta is all equally physical, nor are they all completely compatible with Dirac's theory. It is evident that the GM (3) includes no free parameters so that the momenta (6)-(8) can never be all true. One purpose of this study is to point out that with the complete utilization of Dirac's theory, only one case among (6)-(8) survives, which is even fundamental to the orbital angular momentum therefore the canonical momentum $p_{\varphi}=-i \hbar \partial_{\varphi}$ turns out to be meaningful, but another canonical momentum $p_{\theta}=-i \hbar\left(\partial_{\theta}+\cot \theta / 2\right)$ on the sphere proves to be problematic in physics. However, as a mathematical symbol without any physical meaning preassigned, the quantity $p_{\theta}$ may still be useful.

This paper is organized as follows. In section II, we present a list of five different forms of the GM (6)-(8) proposed by different authors. In section III, we point out that only one form of them is completely compatible with the framework of Dirac's theory, and thus offers 
a proper description of the momentum for the problem under consideration. The commutator between $p_{\theta}$ and the Hamiltonian $H$ as $\left[p_{\theta}, H\right]$ breaks Dirac's canonical quantization procedure, meaning that $p_{\theta}$ is improper. In section IV, it is demonstrated that the GM is fundamental to the orbital angular momentum, and three components of the GM form the generators of a dynamic group on the surface. The self-adjointness of the GM is also discussed in this section. Conclusions and discussions are presented in section V.

\section{GEOMETRIC MOMENTA FOR A PARTICLE ON THE SPHERE: A REVIEW}

For $S^{2}$ parametrized by

$$
x=r \sin \theta \cos \varphi, y=r \sin \theta \sin \varphi, z=r \cos \theta
$$

there are five forms of the GM in the current literature, and all can be written into following form with two real parameters $(\alpha, \beta)$ :

$$
\begin{aligned}
& p_{(\alpha, \beta) x}=-i \hbar \frac{\partial}{\partial x}=-\frac{i \hbar}{r}\left(\cos \theta \cos \varphi \frac{\partial}{\partial \theta}-\frac{\sin \varphi}{\sin \theta} \frac{\partial}{\partial \varphi}-\alpha \sin \theta \cos \varphi\right)+\frac{\hbar}{r} \beta \sin \theta \cos \varphi \\
& p_{(\alpha, \beta) y}=-i \hbar \frac{\partial}{\partial y}=-\frac{i \hbar}{r}\left(\cos \theta \sin \varphi \frac{\partial}{\partial \theta}+\frac{\cos \varphi}{\sin \theta} \frac{\partial}{\partial \varphi}-\alpha \sin \theta \sin \varphi\right)+\frac{\hbar}{r} \beta \sin \theta \sin \varphi \\
& p_{(\alpha, \beta) z}=-i \hbar \frac{\partial}{\partial z}=-\frac{i \hbar}{r}\left(-\sin \theta \frac{\partial}{\partial \theta}-\alpha \cos \theta\right)+\frac{\hbar}{r} \beta \cos \theta .
\end{aligned}
$$

It seems that the corresponding Hamiltonian is then

$$
H=\frac{1}{2 \mu}\left(p_{(\alpha, \beta) x}^{2}+p_{(\alpha, \beta) y}^{2}+p_{(\alpha, \beta) z}^{2}\right)=\frac{L^{2}}{2 \mu r^{2}}+\frac{\hbar^{2}}{2 \mu r^{2}} \gamma
$$

where

$$
\gamma=(\alpha-i \beta)(2-\alpha+i \beta)
$$

It is worth stressing that because of the presence of the notorious operator order problem $^{32,56}$, there is no simple connection between the classical Hamiltonian $H=p^{2} /(2 \mu)$ and the quantum Hamiltonian $H=L^{2} /\left(2 \mu r^{2}\right)$. It is therefore understandable that how to identify the quantum Hamiltonian for particles moving on two-dimensional sphere has been an intriguing problem. To tackle it, more than four proposals have been put forward, including two types of group quantization ${ }^{31,32,57}$, the confining potential procedure ${ }^{35,36}$, and converting the second-class constraints into the first class ones or their equivalent ${ }^{10,56,58-60}$. All lead to the correct one

$$
H=\frac{L^{2}}{2 \mu r^{2}} .
$$


As we will discuss in section III, Dirac's theory itself implies a self-consistent and insightful way to determine the correct form, but it is not simply putting parameters $\beta=0$ and $\alpha=0$ or 2 into (9) to make it go over to (11). As we see shortly, momenta with these two sets of parameters are inconsistent with Dirac's theory.

The following is a list of various forms of the GM in the current literature. Because (6)-(8) give the most general GM of $p_{(\alpha, \beta) i}$ satisfying the fundamental commutators $(20)-(22)$, the differences between them occur in the matter of choice of the parameters of $(\alpha, \beta)$. The GM was first mentioned in 1968 by Gyorgyi and Kovesi-Domokos ${ }^{13}$, who, while investigating the relation between Schwinger's angular momentum calculus and the Dirac bracket, mentioned that, on the two-dimensional sphere, the momentum operator $\mathbf{p}$ acting on the spherical harmonics must take the form $\mathbf{p}=-i \hbar(\nabla-\mathbf{n} / r)$ rather than $\mathbf{p}=-i \hbar \nabla$ itself. This first appearance of the momentum operator happened to be complete and it is evidently a special case of the general form (3), but unfortunately it went unnoticed in all later and relevant studies of the momentum ${ }^{14-30}$.

(i) The first systematic study of the GM was given by Falck and Hirshfeld in 1983, who found $\alpha=2$ and $\beta=0^{14}$.

(ii) Later in 1985, another form of GM was put forward by Schnitzer who set $\alpha=0$ or 1 and $\beta=0^{15}$.

(iii) In 1992, Ikegami, Nagaoka, Takagi and Tanzawa proposed $\alpha=1$ and $\beta \neq 0^{16}$.

(iv) In 2000, Hong, Kim and Park suggested that two real parameters $(\alpha, \beta)$ should be $\alpha= \pm 1$ and $\beta=0^{18}$.

(v) The fifth choice is made by many groups based on different theoretical grounds and it should be $\alpha=1$ and $\beta=0^{13,17,19-23,30}$.

How do we fix the constants $\alpha$ and $\beta$ ? The primary reason must be a physical one: The two sets of fundamental relation (23) and (24) must be imposed upon the form of GM. As a consequence, we have only one choice: $\alpha=1$ and $\beta=0$, which coincides with (3), and $\left[p_{\theta}, H\right](36)$ turns out to be inconsistent with the Dirac's theory. The secondary consideration may come from mathematics: None of these previous approaches (i-v) above have taken the self-adjointness of the GM into serious consideration, and if considered, only the case with $\alpha=1$ and $\beta=0$ is satisfactory again. By a self-adjoint operator, we mean that all its eigenvalues are real and that eigenfunctions corresponding to distinct eigenvalues are orthogonal. In this sense, the well-known canonical momentum $p_{\theta}$ is not a self-adjoint 
operator, as pointed out many years ago ${ }^{61}$.

\section{COMPLETE DETERMINATION OF GEOMETRIC MOMENTA AND THE HAMILTONIAN WITHIN DIRAC'S THEORY}

For a particle on the sphere with radius $R$ in $R^{3}$, the primary constraint is $\varphi_{1}$,

$$
\varphi_{1}=\vec{x}^{2}-r^{2} \approx 0
$$

hereafter the symbol $\approx$ implies a weak equality that is valid on the constraint surface ${ }^{62}$. The secondary constraint $\varphi_{2}$ is given by

$$
\varphi_{2}=\vec{x} \cdot \vec{p} \approx 0
$$

There are, in fact, third and forth constraint within the consistency from the calculation of the Poisson bracket $\left\{\varphi_{j}, H_{p}\right\}_{D} \sim \varphi_{j+1}(j=1,2,3)$, where primary Hamiltonian $H_{p}=$ $p^{2} /(2 \mu)+\lambda\left(\vec{x}^{2}-r^{2}\right)+\dot{\lambda} p_{\lambda}$, where $\lambda$ is the Lagrangian multiplier and $p_{\lambda}$ is its conjugate variable $^{14}$. The last two constraints $\varphi_{3}=p^{2}-2 \lambda m r^{2} \approx 0$ and $\varphi_{4}=\dot{\lambda} \approx 0$ are no longer useful in quantum mechanics ${ }^{62}$.

In classical mechanics, with the introduction of the Dirac bracket instead of the Poisson one for the canonical variables $A$ and $B$,

$$
\{A, B\}_{D} \equiv\{A, B\}-\left\{A, \varphi_{\alpha}\right\} C_{\alpha \beta}^{-1}\left\{\varphi_{\beta}, B\right\}
$$

where $\varphi_{\alpha}(\alpha=1,2)$ are, respectively, the primary and second-class constraint (12) and (13) and the matrix elements $C_{\alpha \beta}$ is defined by

$$
C_{\alpha \beta}=\left\{\varphi_{\alpha}, \varphi_{\beta}\right\}
$$

the primary Hamiltonian $H_{p}$ must then be replaced by the usual one: $H_{p} \rightarrow H$,

$$
H=\frac{p^{2}}{2 \mu}
$$

The positions $x_{i}$ and momenta $p_{i}$ satisfy the following Dirac brackets,

$$
\left\{x_{i}, x_{j}\right\}_{D}=0,\left\{x_{i}, p_{j}\right\}_{D}=\delta_{i j}-\frac{x_{i} x_{j}}{r^{2}},\left\{p_{i}, p_{j}\right\}_{D}=-\frac{1}{r^{2}}\left(x_{i} p_{j}-x_{j} p_{i}\right),
$$


where other Dirac brackets between $x_{i}$ and $p_{j}$ vanish. The equation of motion is in general

$$
\dot{f}=\{f, H\}_{D},
$$

from which we have for $x_{i}$ and $p_{i}{ }^{62}$

$$
\dot{x}=\left\{x_{i}, H\right\}_{D}=\frac{p_{i}}{\mu}, \dot{p}_{i}=\left\{p_{i}, H\right\}_{D}=-\frac{x_{i} p^{2}}{\mu r^{2}} .
$$

We are ready to construct commutator $[A, B]$ of two variables $A$ and $B$ in quantum mechanics, which is attainable by direct correspondence of the Dirac brackets as $[A, B] /(i \hbar) \rightarrow$ $\{A, B\}_{D}$. There are evidently two categories of the fundamental commutator, corresponding to (17) and (19) respectively. The first category comprises commutator between operators $x_{i}$ and $p_{i}$, given by the quantization of Dirac brackets (17):

$$
\begin{aligned}
& {\left[x_{i}, x_{j}\right]=0,} \\
& {\left[x_{i}, p_{j}\right]=i \hbar\left(\delta_{i j}-\frac{x_{i} x_{j}}{r^{2}}\right),} \\
& {\left[p_{i}, p_{j}\right]=-\frac{i \hbar}{r^{2}}\left(x_{i} p_{j}-x_{j} p_{i}\right) .}
\end{aligned}
$$

There is no operator ordering problem in the right-hand side of Eq. (22) because the commutator must satisfy the Jacobian identity. These commutators form a closed algebra, even nonlinear. Given the two-dimensional spherical surface under consideration, there is a family of realizations for this algebra, and one can verify that operators $p_{(\alpha, \beta) i}(6)-(8)$ solve equations (20 )-(22). The second category of the fundamental commutators is given by quantization of (19),

$$
\begin{aligned}
& {\left[x_{i}, H\right]=i \hbar \frac{p_{i}}{\mu},} \\
& {\left[p_{i}, H\right]=-i \hbar \frac{x_{i} H+H x_{i}}{r^{2}} .}
\end{aligned}
$$

Here, in Dirac's quantization from the Dirac bracket $\left\{p_{i}, H\right\}_{D}=-2 x_{i} H / r^{2}$ (19) to the quantum commutator (24), we take the symmetric average of the noncommuting factors $x_{i}$ and $H$. A complete utilization of Dirac's theory means that operators $\left(x_{i}, p_{i}, H\right)$ are necessarily compatible with these two categories of fundamental commutators (20)-(24). A reasonable question arises: Can these commutators be sufficient to result in the unique forms of both the GM and the Hamiltonian? In the general case, the answer is no because the right-hand side of Eq. (24) is by no means unique in quantum mechanics. However, the 
answer to the problem on the two-dimensional surface is yes. To see this, we start from a quite general form of the self-adjoint Hamiltonian $H_{q}$ as

$$
H_{q}=-\frac{\hbar^{2}}{2 \mu} \nabla^{2}+f(\theta, \varphi) \frac{\partial}{\partial \theta}+g(\theta, \varphi) \frac{\partial}{\partial \varphi}+h(\theta, \varphi),
$$

where $f(\theta, \varphi), g(\theta, \varphi)$ and $h(\theta, \varphi)$ are three functions whose explicit forms are to be determined. Substitution of this $H_{q}$ into Eq. (23) results in,

$$
\begin{aligned}
& {\left[x, H_{q}\right]-i \hbar \frac{p_{(\alpha, \beta) x}}{\mu}=(1-\alpha+i \beta) \frac{\hbar}{\mu} \frac{x}{r}+r[-\cos \theta \cos \varphi f(\theta, \varphi)+\sin \theta \sin \varphi g(\theta, \varphi)],} \\
& {\left[y, H_{q}\right]-i \hbar \frac{p_{(\alpha, \beta) y}}{\mu}=(1-\alpha+i \beta) \frac{\hbar}{\mu} \frac{y}{r}+r[\cos \theta \sin \varphi f(\theta, \varphi)+\sin \theta \cos \varphi g(\theta, \varphi)],} \\
& {\left[z, H_{q}\right]-i \hbar \frac{p_{(\alpha, \beta) z}}{\mu}=(1-\alpha+i \beta) \frac{\hbar}{\mu} \frac{z}{r}+r \sin \theta f(\theta, \varphi) .}
\end{aligned}
$$

The terms on the right-hand sides of these equations must be all zero; otherwise, relation (23) as $\left[x_{i}, H\right]=i \hbar p_{(\alpha, \beta) i} / \mu$ will be violated. The only solution is simply

$$
\alpha=1, \beta=0, f(\theta, \varphi)=g(\theta, \varphi)=0 .
$$

The only undetermined function $h(\theta, \varphi)$ in $H_{q}(25)$ can be fixed by use of any one of three commutators (24), and the solution is

$$
h(\theta, \varphi)=0 .
$$

Finally, we not only reproduce the Hamiltonian (11) but also get a unique choice for the GM that can be simply denoted by $\left(p_{x}, p_{y}, p_{z}\right)$,

$$
\begin{aligned}
& p_{x}=-i \hbar\left(\cos \theta \cos \varphi \frac{\partial}{\partial \theta}-\frac{\sin \varphi}{\sin \theta} \frac{\partial}{\partial \varphi}-\sin \theta \cos \varphi\right), \\
& p_{y}=-i \hbar\left(\cos \theta \sin \varphi \frac{\partial}{\partial \theta}+\frac{\cos \varphi}{\sin \theta} \frac{\partial}{\partial \varphi}-\sin \theta \sin \varphi\right), \\
& p_{z}=i \hbar\left(\sin \theta \frac{\partial}{\partial \theta}+\cos \theta\right) .
\end{aligned}
$$

This offers a proper momentum description for a free particle on a two-dimensional sphere.

Can a pair of the well-known canonical momenta $\left(p_{\theta}, p_{\varphi}\right)$ be considered proper? The answer is negative. Application of Dirac's theory to two angular variables $(\theta, \varphi)$ and generalized momenta $\left(p_{\theta}, p_{\varphi}\right)$ gives rise to nonvanishing relevant Dirac brackets:

$$
\begin{gathered}
\{\theta, H\}_{D}=\frac{p_{\theta}}{\mu r^{2}},\{\varphi, H\}_{D}=\frac{p_{\varphi}}{\mu(r \sin \theta)^{2}}, \\
\left\{p_{\theta}, H\right\}_{D}=\frac{\cot \theta}{\mu(r \sin \theta)^{2}} p_{\varphi}^{2}, \quad\left\{p_{\varphi}, H\right\}_{D}=0 .
\end{gathered}
$$


The quantum commutators are simultaneously determined by $\{A, B\}_{D} \rightarrow[A, B] /(i \hbar)$. From two commutators $p_{\theta}=\mu r^{2}[\theta, H] /(i \hbar)$ and $p_{\varphi}=\mu(r \sin \theta)^{2}[\varphi, H] /(i \hbar)$, we immediately arrive at the operators $p_{\theta}=-i \hbar\left(\partial_{\theta}+\cot \theta / 2\right)$ and $p_{\varphi}=-i \hbar \partial_{\varphi}$. Most importantly, we have to know which equations of motion they obey. The result shows $\left[p_{\varphi}, H\right]=0$ fulfills all principles, but another commutator $\left[p_{\theta}, H\right]$, given by

$$
\left[p_{\theta}, H\right]=i \hbar \frac{\cot \theta}{\mu(r \sin \theta)^{2}}\left(p_{\varphi}^{2}-\frac{\hbar^{2}}{4}\right),
$$

violates the correspondence: $\left[p_{\theta}, H\right]\left(\rightarrow i \hbar\left\{p_{\theta}, H\right\}_{D}\right)=i \hbar \cot \theta p_{\varphi}^{2} / \mu(r \sin \theta)^{2}$. So we see that the canonical momentum $p_{\theta}$ breaks Dirac's canonical quantization procedure, and we can safely conclude that it is not a proper momentum for the free particle on the two-dimensional sphere.

\section{GEOMETRIC MOMENTA AS THE GENERATORS OF THE LORENTZ GROUP SO $(3,1)$}

This section will show that the orbital angular momentum $L_{k}$ originally defined by $\varepsilon_{i j k} L_{k} \equiv x_{i} p_{j}-x_{j} p_{i}$ is the consequence of the commutators between momentum components $\left[p_{i}, p_{j}\right]$, and will give an interpretation of $p^{2}$ in relation (9) based on the dynamic group. For further demonstration of the fact that the GM is proper, we will discuss the self-adjointness of the GM (31)-(33) at end of the section.

With help of the transformations $x_{i} \rightarrow x_{i} / r$ and $p_{i} \rightarrow p_{i} / r$, which render the position dimensionless and cause the momentum to be a dimension of Planck's constant $\hbar$, the fundamental (nontrivial) commutators (20)-(22) become

$$
\begin{aligned}
{\left[x_{i}, x_{j}\right] } & =0 \\
{\left[x_{i}, p_{j}\right] } & =i \hbar\left(\delta_{i j}-x_{i} x_{j}\right), \\
{\left[p_{i}, p_{j}\right] } & =-i \hbar \varepsilon_{i j k} L_{k} .
\end{aligned}
$$

We can easily verify the following secondary commutation relations:

$$
\begin{aligned}
{\left[L_{i}, p_{j}\right] } & =i \hbar \varepsilon_{i j k} p_{k}, \\
{\left[L_{i}, x_{j}\right] } & =i \hbar \varepsilon_{i j k} x_{k}, \\
{\left[L_{i}, L_{j}\right] } & =i \hbar \varepsilon_{i j k} L_{k} .
\end{aligned}
$$


Other commutation relations between $L_{i}, x_{i}$ and $p_{i}$ are zero.

Clearly, these commutators (37)-(42) between ten operators $\left\{1, x_{i}, p_{i}, L_{i}\right\}$ form a closed algebra. However, it contains an equation (38) whose right-hand side is nonlinear in terms of generator $x_{i}$. So the algebra is associated with a nonlinear $S O(3,2)$ group. We leave this nonlinear group for further studies. Moreover, we can easily identify two familiar subgroups: one is Lorentz group $S O(3,1)$ whose six generators are $\left\{p_{i}, L_{i}\right\}$ satisfying commutators (39), (40), and (42), and another is the usual rotational group $S O(3)$ whose three generators are three Cartesian components of the angular momentum $L_{i}$, defined by (42) alone. Even the nonlinear $S O(3,2)$ group is a little bit strange, the Lorentz group $S O(3,1)$ is elementary for theoretical physicists and the GM $p_{i}$ acts as the "boost" that a vital part of group $S O(3,1)$ in our research. Note that the two infinitesimal "boosts" $\delta \psi$ and $\delta \phi$ along the $x$ and $y$ directions involve a rotation $\delta \psi \delta \phi$ about the $z$-axis,

$$
\begin{aligned}
& \exp \left(i \delta \psi p_{x}\right) \exp \left(i \delta \phi p_{y}\right) \exp \left(-i \delta \psi p_{x}\right) \exp \left(-i \delta \phi p_{y}\right) \\
& =1-\left[p_{x}, p_{y}\right] \delta \psi \delta \phi+\ldots \\
& =1+i \hbar L_{z} \delta \psi \delta \phi+\ldots
\end{aligned}
$$

This implies that three GM operators $p_{i}$ are generators of the infinitesimal parallel transport of a vector on the surface, which will be studied in detail elsewhere.

Two Casimir operators of Lorentz group $S O(3,1)$ are

$$
\begin{aligned}
& C_{1} \equiv L_{i} L_{i}-p_{i} p_{i}=-\hbar^{2} / 4, \\
& C_{2} \equiv p_{i} L_{i}=0 .
\end{aligned}
$$

Clearly, quantum Hamiltonian (11) (proportional to $L_{i} L_{i}$ ) is not the direct quantization of the classical Hamiltonian (16) (proportional to $p_{i} p_{i}$ ); they differ in a constant that is one of the Casimir operators of group $S O(3,1)$.

As a consequence of relation (40), the operators $p_{x}$ and $p_{y}$ can be obtained from $p_{z}$ by rotation of the axis's rotation. Explicitly, rotation $\pi / 2$ around the $y$-axis renders $p_{z}$ to be $p_{x}$, and $-\pi / 2$ around the $x$-axis renders $p_{z}$ to be $p_{y}$,

$$
p_{x}=\exp \left(-i \pi L_{y} / 2\right) p_{z} \exp \left(i \pi L_{y} / 2\right), p_{y}=\exp \left(i \pi L_{x} / 2\right) p_{z} \exp \left(-i \pi L_{x} / 2\right) .
$$

Here we follow the convention that a rotation operation affects a physical system itself ${ }^{63}$. Hence the eigenvalue problem for operators $p_{x}$ or $p_{y}$ is simultaneously determined once the 
complete solution to $\hat{p}_{z} \psi_{p_{z}}(\theta)=p_{z} \psi_{p_{z}}(\theta)$ is known, where over operator $p_{z}$ on the left-hand side of this equation the carat is used to distinguish it from the eigenvalue $p_{z}$ on the righthand side. The eigenfunctions form a complete set once the eigenvalues $p_{z}$ are real and continuous,

$$
\psi_{p_{z}}(\theta)=\frac{1}{2 \pi} \frac{1}{\sin \theta} \tan ^{-i p_{z}}\left(\frac{\theta}{2}\right)
$$

They are $\delta$-function normalized,

$$
\begin{aligned}
& \oint \psi_{p^{\prime}}^{*}(\theta, \phi) \psi_{p_{z}}(\theta, \varphi) \sin \theta d \theta d \varphi \\
& =\frac{1}{2 \pi} \int_{0}^{\pi} \exp \left(i\left(p_{z}^{\prime}-p_{z}\right)\left(\ln \tan \frac{\theta}{2}\right)\right) \frac{1}{\sin \theta} d \theta \\
& =\frac{1}{2 \pi} \int_{0}^{\pi} \exp \left(i\left(p_{z}^{\prime}-p_{z}\right) \ln \tan \frac{\theta}{2}\right) d \ln \tan \frac{\theta}{2} \\
& =\frac{1}{2 \pi} \int_{-\infty}^{\infty} \exp \left(i\left(p_{z}^{\prime}-p_{z}\right) z\right) d z \\
& =\delta\left(p^{\prime}{ }_{z}-p_{z}\right)
\end{aligned}
$$

where the variable transformation $\ln \tan \theta / 2 \rightarrow z$ is used. So, we see explicitly that the eigenfunctions $\psi_{p_{z}}(\theta)$ form a complete set. Readers may check that other forms of momentum in (6)-(8) are not the self-adjoint operator.

\section{CONCLUSIONS AND DISCUSSIONS}

In Dirac's canonical quantization theory on systems with second-class constraints, the commutators between the position, momentum and Hamiltonian form a set of algebra relations that are fundamental in the construction of both the quantum momentum and the Hamiltonian. For the particle moving on a spherical surface or the rotation of a spherical top, it gives rise to profound consequences. Some of them are in the following. (i) The wellknown canonical momentum operator $p_{\theta}$ represents no physical quantities because it breaks Dirac's canonical quantization procedure, and the proper momentum is obtainable from an extrinsic description in flat space rather than an intrinsic one within the surface itself. (ii) Three nontrivial commutators between components of the GM generate three components

of the orbital angular momentum. So another well-known canonical momentum operator $p_{\varphi}$ is meaningful and actually represents the $z$-component of the angular momentum $L_{z}$. (iii) 
The components of angular momentum and GM are six generators of the Lorentz $S O(3.1)$ group, where the GM acts as the boost.

The five different forms of the GM in the current literature are revisited, and only one of them is shown to be self-adjoint and completely compatible with Dirac's theory. Dirac's canonical quantization theory for the second-class constraints is more insightful than it used to seem to be, and one must use it with great care. As far we can see today, among all previous publications including some by the current authors and possibly this one, "Some are correct; some are less correct. Some have original features; some are less rich in this praiseworthy property" ${ }^{4}$. In any case, we hope this contribution to the literature will be a valuable one.

\section{ACKNOWLEDGMENTS}

This work is financially supported by National Natural Science Foundation of China under Grant No. 11175063, and by the Program for New Century Excellent Talents in University, Ministry of Education, China. One of the authors (Q. H. Liu) would like to thank Professor H. J. Zhou for his kind hospitality during 1-21, August 2011, at the Institute of Theoretical Physics (ITP), CAS, Beijing, where the revision was completed, and for Professors Z. C. Ouyang and W. M. Zheng at ITP for their kind interest in this work.

* quanhuiliu@gmail.com

1 P. A. M. Dirac, Lectures on quantum mechanics (Yeshiva University, New York, 1964); Can. J. Math. 2, 129(1950).

2 H. Grundling, Rep. Math. Phys., 57, 97(2006); H. Grundling, C. A. Hurst, J. Math. Phys. 39, 3091(1998).

3 H. J. Rothe and K. D. Rothe, Classical and Quantum Dynamics of Constrained Hamiltonian Systems, (World Scientific, Singapore, 2010).

4 L. S. Schulman, Techniques and Applications of Path Integration, (Wiley, New York, 1981).

5 M. Henneaux and C. Teitelboim, Quantization of Gauge Systems (Princeton University Press, Princeton, NJ, 1992). 
6 A. A. Kirilov, Geometric Quantization, in Dynamical Systems IV: Symplectic Geometry and its Applications, (Encyclopaedia Math. Sci. IV) ed. V. I. Arnold and S. I. Novikov (Springer, NY, 1990) P.139; N. M. J. Woodhouse, Geometric Quantization (Clarendon, Oxford, 1992).

7 P. A. M. Dirac, The Principles of Quantum Mechanics, 4th ed. (Oxford University Press, Oxford, 1967) P.114.

8 P. Maraner, Ann. Phys. 246, 325(1996).

9 L. Kaplan, N. T. Maitra and E. J. Heller, Phys. Rev. A 56, 2592(1997).

10 J. R. Klauder, S. V. Shabanov, Nucl. Phys. B 511, 713(1998).

11 D. Baleanu and Y. Güler, J. Phys. A: Math. Gen. 34, 73(2001).

12 A. G. Nuramatov, l. V. Prokhorov, Int. J. Geom. Meth. Mod. Phys. 3, 1459(2006).

13 G. Gyorgyi and S. Kovesi-Domokos, IL Nuovo Cimento B 58, 191(1968).

14 N. K. Falck and A. C. Hirshfeld, Eur. J. Phys. 4, 5(1983).

15 H. J. Schnitzer, Nucl. Phys. B 261, 546572(1985).

16 M. Ikegami and Y. Nagaoka, S. Takagi and T. Tanzawa, Prog. Theoret. Phys. 88, 229(1992).

17 S. Ishikawa, T. Miyazaki, K. Yamamoto, M. Yamanobe, Int. J. Mod. Phys. A, 11, 3363(1996).

18 S. T. Hong, W. T. Kim and Y. J. Park, Mod. Phys. Lett. A 15, 1915(2000).

19 T. Homma, T. Inamoto, T. Miyazaki, Phys. Rev. D 42, 2049(1990); Z. Phys. C 48, 105(1990).

20 Q. H. Liu, and T. G., Liu, Int. J. Theor. Phys. 42, 2877(2003).

21 A. V. Golovnev, Int. J. Geom. Meth. Mod. Phys. 3, 655(2006).

22 Q. H. Liu, C. L. Tong and M. M. Lai, J. Phys. A: Math. and Theor. 40, 4161(2007).

23 A. V. Golovnev, Rep. Math. Phys. 64, 59(2009).

24 Q. H. Liu, J. X. Hou, Y. P. Xiao and L. X. Li, Int. J. Theor. Phys. 43, 1011(2004).

25 Q. H. Liu, Int. J. Theor. Phys. 45, 2167(2006).

26 X. Wang, Y. P. Xiao, T. G., Liu, M. M. Lai, and J. Rao, Int. J. Theor. Phys. 45, 2509(2006).

27 M. M. Lai, X. Wang, Y. P. Xiao and Q. H. Liu, Commun. Theor. Phys. 46, 843(2006).

28 Y. P. Xiao, M. M. Lai, J. X. Hou, X. W. Chen and Q. H. Liu, Commun. Theor. Phys. 44, 49(2005).

29 Q. H. Liu, Acta Physics Sinica 57, 674(2008).

30 X. M. Zhu, M. Xu, and Q. H. Liu, Int. J. Geom. Meth. Mod. Phys. 3, 411(2010).

31 P. W. Higgs, J. Phys. A: Math. Gen. 12, 309(1979). This classic work does not have terminology such as group quantization or group correspondence principle, but he obtain the correct quantum 
mechanical Hamiltonian $H_{o}$ for a free particle on a sphere $S^{N}$ from its classical counterpart by substituting for the classical Casimir operator of the geometrical $S O(N+1)$ group its quantum mechanical counterpart, with emphasizing that "The requirement of symmetry removes ordering ambiguities other than those which would give rise to an unobservable additive constant in $H_{o}$ ". H. Kleinert, Path Integrals in Quantum Mechanics, Statistics, Polymer Physics, and Financial Markets, 5th ed., (World Scientific, Singapore, 2009).

33 H. Kleinert, Multivalued Fields in Condensed Matter, Electromagnetism, and Gravitation, (World Scientific, Singapore, 2008).

34 A. Karamatskou and H. Kleinert, arXiv:1102.2486v1.

35 H. Jensen and H. Koppe, Ann. Phys. 63, 586(1971).

36 R. C. T. da Costa, Phys. Rev. A 23, 1982(1981).

37 A. Szameit, et. al, Phys. Rev. Lett. 104, 150403(2010).

38 G. Cantele, D. Ninno, and G. Iadonisi, Phys. Rev. B 61, 13730 (2000).

39 H. Aoki, M. Koshino, D. Takeda, H. Morise, and K. Kuroki, Phys. Rev. B 65, 035102 (2001).

40 M. Encinosa and L. Mott, Phys. Rev. A 68, 014102 (2003).

41 N. Fujita and O. Terasaki, Phys. Rev. B 72, 085459 (2005).

42 M. Koshino and H. Aoki, Phys. Rev. B 71, 073405 (2005).

43 J. Gravesen and M. Willatzen, Phys. Rev. A 72, 032108 (2005).

44 A. V. Chaplik and R. H. Blick, New J. Phys. 6, 33 (2004).

45 A. Marchi, S. Reggiani, M. Rudan, and A. Bertoni, Phys. Rev. B 72, 035403 (2005).

46 G. Ferrari and G. Cuoghi, Phys. Rev. Lett. 100, 230403 (2008).

47 B. Jensen and R. Dandoloff, Phys. Rev. A 80, 052109 (2009); Phys. Rev. A 81, 049905(E) (2010).

48 B. Jensen and R. Dandoloff, Phys. Lett. A 375, 448-451(2011).

49 C. Ortix and J. van den Brink, Phys. Rev. B 81, 165419(2010).

50 C. Ortix and J. van den Brink, Phys. Rev. B 83, 113406 (2011).

51 B. Podolsky, Phys. Rev. 32, 812(1928).

52 L. I. Schiff, Quantum Mechanics, (McGraw-Hill, New York, 1949) p. 135.

53 B. S. DeWitt, Phys. Rev. 85, 653(1952); Rev. Mod. Phys. 29, 377(1957).

54 K. S. Cheng, J. Math. Phys. 13, 1723(1972).

55 H. Dekker, Phys. A 103, 586(1980). 
56 H. Kleinert and S. V. Shabanov, Phys. Lett. A 232, 327(1997).

57 V. Aldaya, M. Calixto, J. Guerrero, F. F. Lopez-ruiz, Rep. Math. Phys. 64, 49(2009); arXiv:1012.3681v1, and references therein.

58 P. Dita, Phys. Rev. A 56, 2574(1997).

59 C. Neves and C Wotzasek, J. Phys. A: Math. Gen. 33, 6447(2000).

60 D. Baleanu, Y. Guler, J. Phys. A: Math. Gen. 34, 73(2001).

61 J. M. Domingos, and M. H. Caldeira,. Found. Phys. 14, 147(1984).

62 As Dirac called, $\varphi_{m} \approx 0$ weak equations, to distinguish them from the usual or strong equations. One can make use of $\varphi_{m}=0$ only after one has worked out all the Poisson brackets. For instance, the third constraint $\varphi_{3}=p^{2}-2 \lambda \mu r^{2} \approx 0$ solves the Lagrangian multiplier $\lambda=$ $p^{2} /\left(2 \mu r^{2}\right)$ which must be used in determining the equation of motion for $\dot{p}_{i}=\left[p_{i}, H_{p}\right]=-2 \lambda x_{i}=$ $-x_{i} p^{2} /\left(\mu r^{2}\right)$. It is identical to $\dot{p}_{i}=\left\{p_{i}, H\right\}_{D}$ where $H=p^{2} /(2 \mu)$ rather than the primary one $H_{p}=p^{2} /(2 \mu)+\lambda\left(\vec{x}^{2}-r^{2}\right)+\dot{\lambda} p_{\lambda}$. In general, with Hamiltonian $H=p^{2} /(2 \mu)$ is used, we must simultaneously use the Dirac bracket in all cases. In quantum mechanics, there is only $H$ that is free from Lagrangian multipliers; and the quantum commutators $[A, B] /(i \hbar)$ must directly correspond to Dirac bracket $\{A, B\}_{D}$.

63 J. J. Sakurai, Modern Quantum Mechanics, Revised Edition (Addison-Wesley, New York. 2005).

\section{About the authors}

Q. H. Liu: M., Ph.D. (1996-1999, ITP; supervisiors: Professor H. W. Peng and Professor Z. C. Ouyang), Professor of Physics (1998-), Director (2003-) of School for Theoretical Physics, Hunan University. Research interests: Quantum mechanics, statistical and condensed matter physics.

L. H. Tang: M., M.S. candidate (2011, expected; supervisior: Professor Q. H. Liu). D. M. Xun: F., Ph.D. candidate (2013, expected; supervisior: Professor Q. H. Liu). 